\title{
Assessment of eggs freshness by means of pulsed infrared thermography
}

\author{
by F. Freni*, A. Quattrocchi*, A. Di Giacomo*, S. A. Piccolo**, R. Montanini*, ${ }^{\star \star \star}$ \\ * Dept. of Engineering, University of Messina, C.da di Dio, 98166 Messina, Italy - Corresponding author: \\ rmontanini@unime.it \\ ** Dept. of Management Engineering, Technical University of Denmark, Lyngby 2800, Denmark \\ *** Institute for Advanced Energy Technologies "Nicola Giordano", CNR-ITAE, 98126 Messina, Italy
}

\begin{abstract}
The paper examines the topic of identifying eggs freshness through an original approach based on pulsed thermography. The proposed method relies on a short thermal stimulation performed by means of a xenon flash, which rises up the temperature of the egg of less than $1{ }^{\circ} \mathrm{C}$. The entire process requires about $1 \mathrm{~s}$, thus providing an effective quality control tool for assessing the freshness of eggs intended for human consumption. The identification and evaluation procedure have been automated by developing a dedicated image processing algorithm. The software performs a series of preliminary operations on the acquired thermograms and returns an estimation of the air chamber's projections, that are correlated to egg degradation and weight reduction.
\end{abstract}

\section{Introduction}

Eggs consumption is regulated by European legislation, setting very restrictive parameters for the conservation and imposing optimum conditions for the shell, cuticula, air cell, yolk, alum, germ, foreign bodies and atypical odours [1]. The air chamber is an important element to determine the eggs freshness; its size is dependent on the egg aging itself. The European Community regulations require that the air chamber height, when the egg is motionless, must not exceed 6 $\mathrm{mm}$, which is reduced to $4 \mathrm{~mm}$ for the eggs indicated like "extra fresh" [2]. During storage, due to aging, the egg $\mathrm{pH}$ increases, the vitvelline and shell membranes undergo a natural deterioration losing consistency, and the albumen decreases its thickness due to the disintegration of ovomucoid mucoprotein. The result is the mixing of albumen organic components and, ultimately, their proteins cleavage, resulting in decreased egg quality [3]. The air chamber is usually very small when the egg is spawned, but during the time the water, contained inside it, evaporates (dehydration). This natural effect favours air penetration through the shell and the shell membrane, producing an enzymatic decomposition process with $\mathrm{CO} 2$ reduction and consequent air chamber growth. These degradation phenomena are accentuated if the storage conditions (temperature and humidity) are particularly adverse [4].

Traditionally, eggs internal inspection takes place through candling and visual observation. The tests exploit a light source (incandescent or led lamp), which is used to show the egg contents in transmission [5]. The yolk must appear like a weak shadow and must not move during a rotation of $180^{\circ}$ along the egg transverse axis. Because of aging, the yolk is free to approach the shell and, consequently, a darker shadow is perceived. The air chamber is placed on the egg bottom (dull pole) and it is visible at candling, especially during its growth [6]. Such methods, however, do not allow a complete quantitative analysis to be performed, but they are limited to subjective qualitative examination. The major limitations are related to human discretion and a slow control procedure [7].

In recent decades, various alternative experimental approaches have been proposed in order to overcome visual inspection limitations. Kemps et al. [8] used visible transmission spectroscopy to estimate the egg freshness by putting it in relation to the $\mathrm{pH}$ level and the albumen quality. The application of this kind of survey involves overlapping and combining different spectrum bands. This is due to a significant decrease in selectivity, producing spectral peaks of difficult interpretation. The aforementioned limits can be overwhelmed by working in the near infrared field, as shown by Schmilovitch et al. [9]. The authors monitored egg samples for 28 days, identifying air chamber size, number of days postbroiling, weight reduction, and $\mathrm{pH}$ levels with correlation coefficients between 0.90 and 0.92 . However, the presence of numerous compounds often makes difficult to individually identify their characteristics [10]. Moreover, water, naturally present in eggs, is absorbed in the near infrared and this may greatly affects the test accuracy [11]. Posudin [12] proposed to apply fluorescence spectroscopy to analyse egg freshness. He found that fresh egg self-fluorescence is ever greater than that of an older one, since it is closely related to the porphyrin level. Recently, Giunchi et al. [13] developed a prediction model of egg freshness based on data obtained by FT-NIR spectroscopy. All spectroscopy techniques guarantee a rapid analysis and the simultaneous evaluation of numerous parameters. In any case, given the high number of parameters of influence and the difficult interpretation of the results, spectroscopy has not adequately taken root in the field of quality control of egg production for human [14].

A totally different approach is that proposed by Völgyi [15], who developed an experimental apparatus based on microwave attenuation, measuring water reduction and egg cross-section. However, this is a preliminary work and the few reported data do not allow for a complete evaluation of the method effectiveness.

Other experiments are aimed to define the egg freshness through the use of an electronic nose. Dutta et al. [16] developed a system consisting of 4 commercially available tin oxide sensors able to detect the freshness of two eggs categories (intact and with small holes on the shell) for aging of 20 to 40 days under weakly controlled storage conditions 
(temperature of $25 \pm 15^{\circ} \mathrm{C}$ and relative humidity of $30 \pm 10 \%$ ). The obtained results, processed by a neural network, provide $95 \%$ of accuracy, but no details on the analysed chemical compounds were provided. Yongwei et al. [17] conducted a similar study investigating a sample of 400 eggs, divided into two categories, stored at a temperature of $22 \pm 1^{\circ} \mathrm{C}$ and refrigerated at $4^{\circ} \mathrm{C}$ for 4 weeks. The authors used a matrix with eight gas sensors to produce a freshness prediction model focused on the albumen quality indicator (HU, Haugh Unit, Raymond Haugh, 1937) and the yolk factor, obtaining a standard prediction error of 3.74 and 0.02 and a correlation coefficient of 0.91 and 0.93 respectively. However, results have not been reported for individual units and only aggregated data were displayed.

Aboonajmi et al. [18] applied ultrasounds: the phase velocity was put in relation to HU and the air chamber height, while the mean amplitudes of the signal peaks were used to estimate egg aging. The study was conducted for 5 weeks on two sample groups, at $24 \pm 1{ }^{\circ} \mathrm{C}$ and $5 \pm 1{ }^{\circ} \mathrm{C}$, with relative humidity of $75 \%$ and $40 \%$ respectively. Although the results are favourable, the technique is based on the consideration, which is not always valid and generalizable, to have a uniform and similar shell for all the analysed eggs.

In the present paper, for the first time, a method based on pulsed infrared thermography [19-20] is proposed. Through this technique is possible to easily visualize, non-invasively and without altering the organoleptic egg's properties, the internal air cell. Since the air chamber size is linked to the egg's freshness, this technique provides a powerful quantitative analysis tool for quickly assessing the eggs conservation status for human consumption. The evaluation procedure has been automated through a dedicated data processing algorithm based on a morphologic operator with white top hat transformation. The algorithm performs a series of preliminary operations on the individual batches of acquired thermograms and returns an estimation of the air chamber projection areas.

\section{Materials and Methods}

\subsection{Experimental approach}

The proposed survey methodology is based on an active thermography approach by means of pulsed thermal stimulation in reflection. The thermal wave, obtained using a $3 \mathrm{~kJ}$ xenon toroidal flash, produces a small overheating of the egg (less than $3{ }^{\circ} \mathrm{C}$ ) for a duration that is typically $10 \mathrm{~ms}$. This gives rise to a thermal front which propagates within the egg. Due to the different density of the air with respect to the liquid content of the egg, a local alteration of the thermal diffusivity is determined, so that the ("defect") air chamber appears as an area of different temperature with respect to the surrounding area. The air contained inside the egg undergoes greater heating than the remaining part and can therefore be easily identified by the infrared camera.

The experimental setup (Fig. 1) consisted of a high-resolution infrared (IR) camera, a pulsed thermal source (flash), and a pulse/acquisition synchronization unit. The IR camera (FLIR SC7600, NETD < 20 mK at room temperature), having a resolution of $640 \times 512$ pixels, works in the medium infrared range (MWIR 3.6-5.1 $\mu \mathrm{m})$. The camera was equipped with a $50 \mathrm{~mm}$ lens and placed at a distance of about $40 \mathrm{~cm}$ from the egg middle plane on an optical table. The toroidal flash was interposed between the camera and the egg, as to ensure a sufficiently uniform heating of the region of interest. A cylindrical PVC support was glued on each egg to make repositioning of the sample in front of the IR camera reproducible. The support consisted of a ball bearing and two fixed references positioned at $0^{\circ}$ and $90^{\circ}$. The synchronization unit (Edevis $\mathrm{GmbH}$, Stuttgart, Germany) allows to trigger the acquisition with the generated pulse. Thermograms were acquired both during heating and cooling, with sampling frequency of $100 \mathrm{~Hz}$. The total duration of each test is typically $1000 \mathrm{~ms}$.

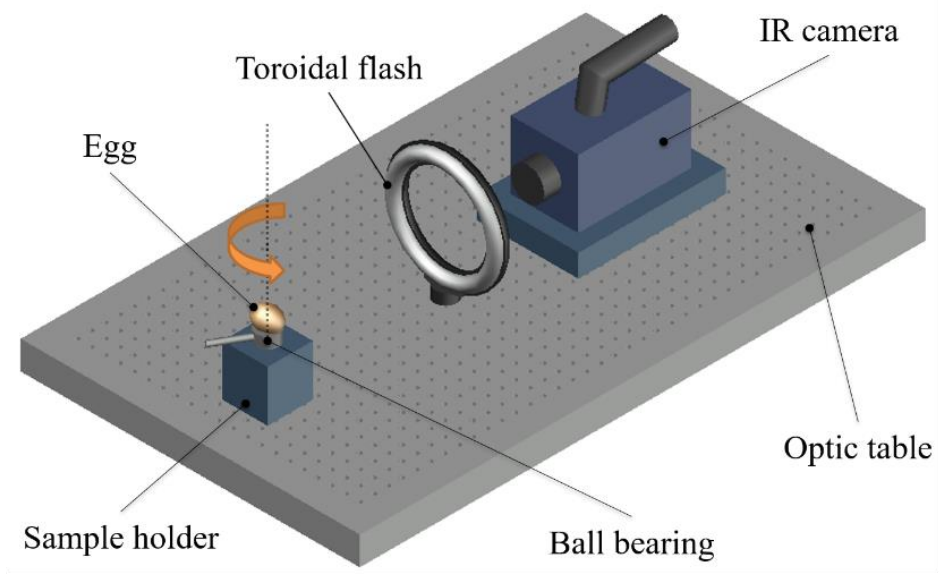

Fig. 1. Schematic drawing of the experimental setup used for assessing egg's freshness by means of the proposed approach based on pulse IR thermography 


\subsection{Testing procedures}

The investigation was carried out using a sample of 18 eggs, divided into three dimensional classes [1, 2] of 6 units each: (XL) extra-large size eggs (weight > $73 \mathrm{~g}$ ); $(\mathrm{L})$ large size eggs (weight $=63-73 \mathrm{~g}$ ); (M) medium size eggs (weight $=53-63 \mathrm{~g}$ ). All eggs had an expiration date of 17 days from the date of purchase. In order to minimize the influence of environmental variables, the test protocol provided for the duration of the experiments the conservation of the eggs within a climatic chamber with a temperature of $28 \pm 0.1^{\circ} \mathrm{C}$ and relative humidity of $30 \pm 0.1 \%$. These climatic conditions, which are more severe than those usually recommended for storage $\left(15-18^{\circ} \mathrm{C}, \mathrm{RH} 40-70 \%\right)$ [2,3], have been deliberately chosen to accelerate the aging of the eggs.

All eggs were tested for 20 days, well beyond their expiration date. At regular intervals, the eggs were removed from the climatic chamber and, after waiting until a surface temperature of $27 \pm 0.2^{\circ} \mathrm{C}$ had been reached, weighed with an analytical balance. Then, the eggs were placed on the sample holder and tested by acquiring the heating and cooling profiles. Finally, two thermograms were recorded at time $t=600 \mathrm{~ms}$, corresponding to the two orthogonal views (front and lateral).

\section{Results and Discussion}

\subsection{Raw IR images}

The following two figures show some raw representative IR images obtained in the following days, frontally to the egg's dull pole (Fig. 2) and laterally to the same (Fig. 3). Day 0 corresponds to the first day of testing, coinciding with the date of purchase. Consequently, the legal expiration of the eggs corresponds to the 17th day of the trial. The three rows of each figure refer to eggs belonging to one of the three commercial categories examined (M, $L$ and $X L)$. The thermograms, in shades of gray tones, all have the same temperature scale.

As expected, the air chamber is always placed on the bottom of the egg (dull pole), has a characteristic shape and is subject to significant growth over time. It is clearly identifiable, with good thermal contrast, from the first day of observation, both in the front and in the lateral images. Tests carried out on freshly deposited fresh eggs (not shown here for reasons of space) indicate that the formation of the air chamber occurs instantaneously when the egg is deposited. The initial position of the air chamber is never perfectly barycentric in the cross section. Its development, produced by the natural dehydration process that acts as a catalyst favouring the enzymatic decomposition processes of the proteins contained in the albumen, is not symmetrical, even if the egg has been kept in a vertical position and has not undergone significant oscillations during the storage time. The geometry appears pseudo-circular in the front view (Fig. 2) and like an ovoid cap in the lateral view (Fig. 3). Therefore, the volume of the air chamber can be geometrically approximated by the sum of two volumes, a spheroid and an ellipsoid.

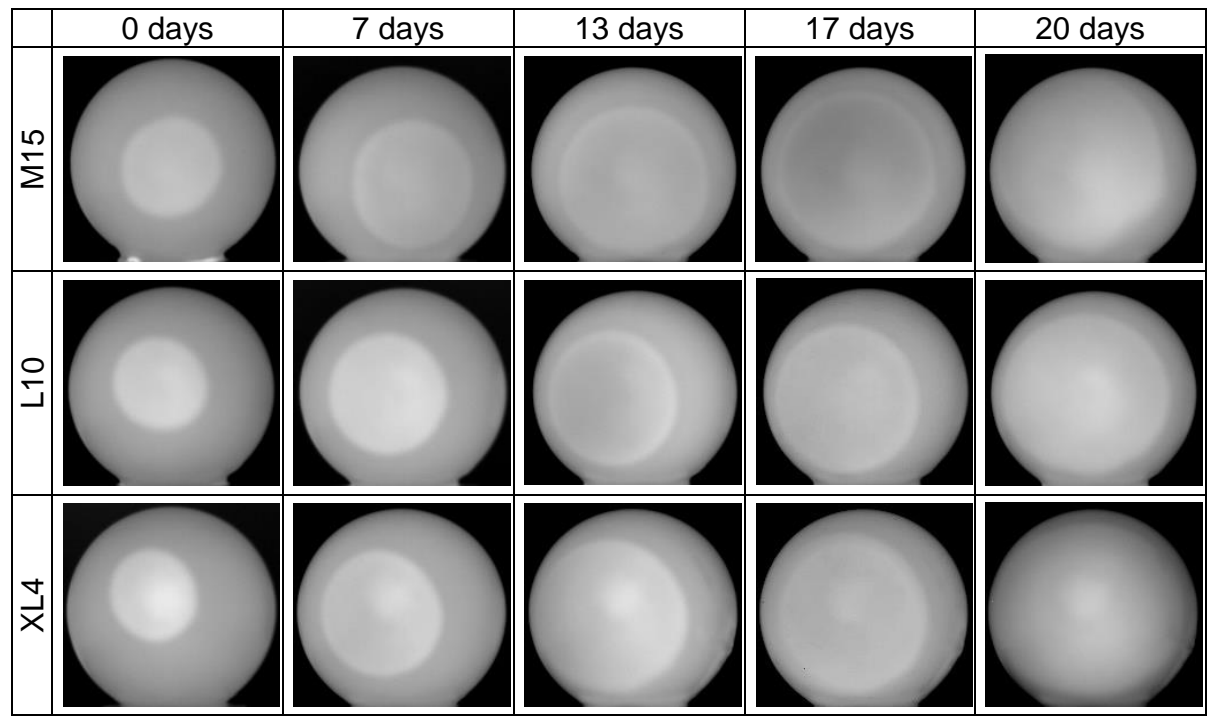

Fig. 2. Examples of raw grayscale IR images taken from egg's dull pole (front view). The alphanumeric code denotes the egg's commercial category ( $\mathrm{M}$ - medium size, $\mathrm{L}$ - large size, $\mathrm{XL}$ - extra-large size) and sample 


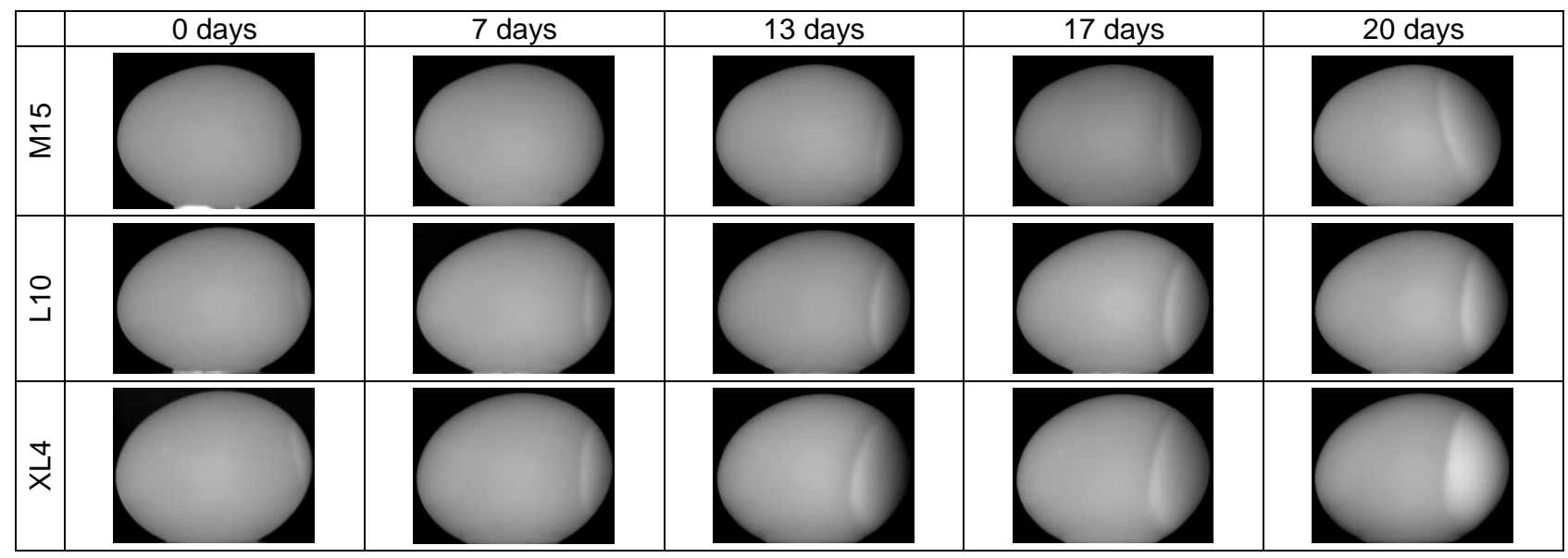

Fig. 3. Examples of raw grayscale IR images taken by rotating the egg by $90^{\circ}$ (lateral view). The alphanumeric code denotes the egg's commercial category ( $M$ - medium size, $L$ - large size, $X L$ - extra-large size) and sample

Finally, it is interesting to observe how the investigation technique developed allows to immediately identify any anomalies in the structure of the egg that is not visible to the naked eye, providing a powerful tool for checking egg's quality. Fig. 4 shows the comparison between two eggs belonging to the same category (L), in which the first one appears sound while the second one presents a corrupted air cell, putting into evidence a partial cleavage of the shell membrane that has been intentionally obtained by shaking strongly the egg. The broken egg does not show any alteration of the outer shell on visual examination, but the projection areas of the camera appear irregular in the infrared and larger than normal. In compliance with the regulations in force [1, 2], these kinds of eggs are not suitable for human consumption.
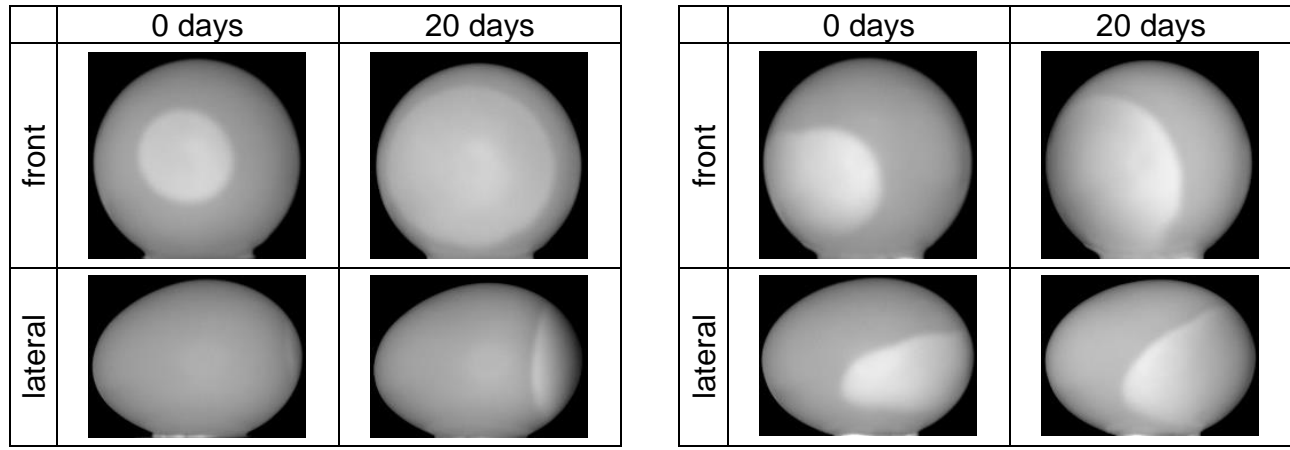

Fig. 4. Raw IR images of a sound egg (left) and of a damaged egg (right)

\subsection{Image processing and automatic sizing}

The raw IR images were post-processed using software specifically developed for the application. Fig. 5 shows the algorithm fundamental phases, in the case of IR images taken in the frontal view.

The original image is captured and the egg is separated from its background which contains the support on which the egg was placed. Subsequently the air chamber area is segmented by the egg's area (in Fig. 5, the air chamber area is related to the egg's area). Air chamber estimation is obtained by converting its image into a binary mask, where the pixels having a value of 1 indicate the area of the air chamber, and counting the number of pixels forming part of the air chamber. The area thus obtained, converted into $\mathrm{mm}^{2}$ after image calibration, can be related to the egg's area, obtaining a percental ratio.

The egg separation from the background was achieved, after employing a Wiener filter to eliminate part of the noise due to reflections, by applying a morphological aperture to the image, using a circle of $100 \times 100$ pixels as structural element. The air chamber segmentation from the rest of the egg is made more complicated by the uneven temperature due to the egg's cooling. This phenomenon produces blurred and/or false edges; for these reasons edge detection segmentation approaches appear ineffective (Prewitt, Roberts, Canny, and Laplacian methods have been tried). 


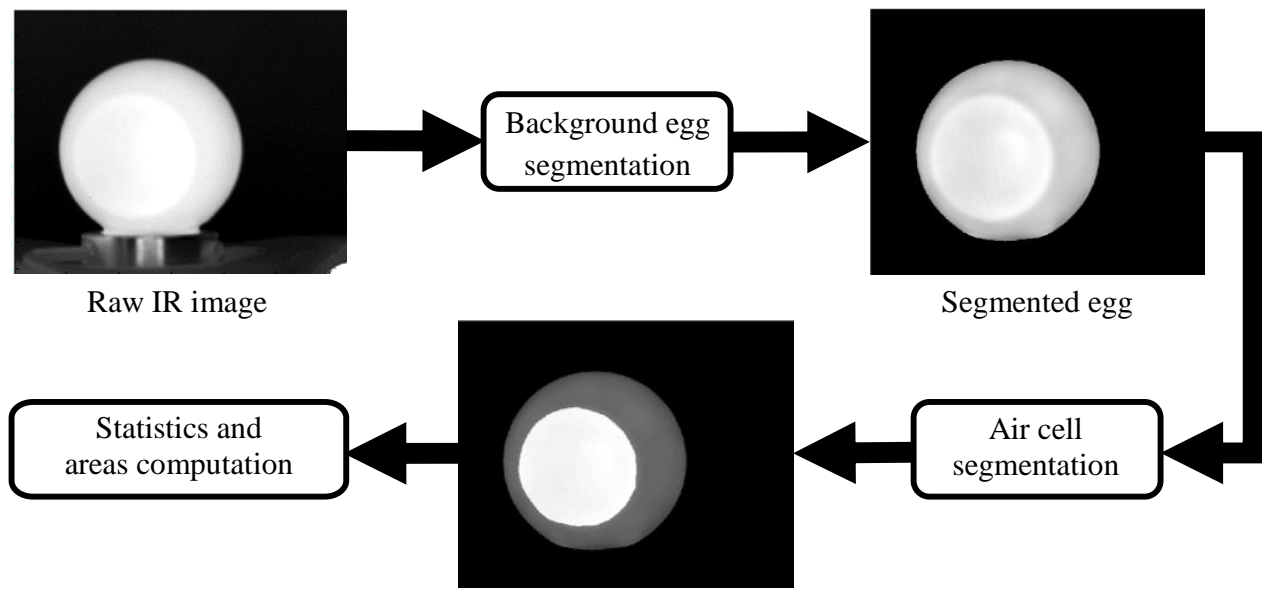

Segmented air cell

Fig. 5. Block diagram of the segmentation approach used to post-process raw IR images taken in the frontal view

The actual air chamber segmentation from the egg area is achieved by computing the top hat operator applied to the segmented egg. The top hat is converted to a binary mask on which morphological opening calculations and morphological closure were applied, using as a structural element a circle of 4 pixels. Any "holes" in the mask are automatically filled and any small area (less than 1000 pixels) erased. Finally, the result is refined by building a convex polygon around the area (Fig. 6).

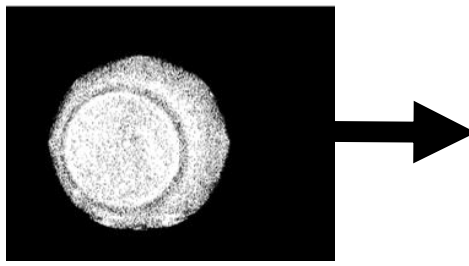

Segmented egg top hat

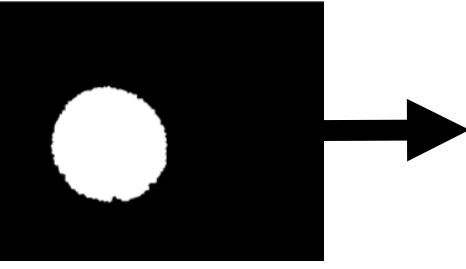

Segmented air cell

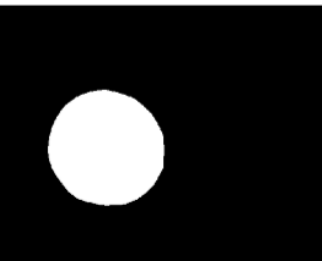

Air cell refinement

Fig. 6. Details of the air chamber segmentation phase (front view)

\subsection{Trend charts}

Over time and due to the dehydration process, eggs undergo heavy weight loss. Fig. 7 shows the mean percentage weight reduction for the tested egg, classified according to commercial size ranges. The axes origin corresponds to the test first day (day 0), which has been used for normalizing the ordinates scale. For all commercial categories, the trend appears linear in first approximation $\left(R^{2}>0.996\right)$, with modest dispersion of the experimental data $\left(S_{y / x}<0.0022\right)$. It is interesting to observe that the slope of the trend line varies according to the class of belonging, resulting more accentuated for the smaller eggs which, therefore, would seem to deteriorate more quickly if we consider weight loss as a symptomatic indicator of aging.

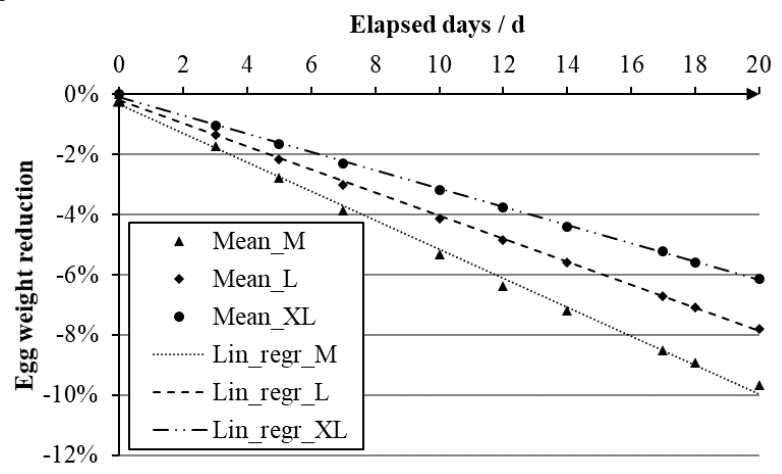

Fig. 7. Reduction of the average percentage weight per class of belonging of the eggs: $M$ - medium size eggs, $L$ - large size eggs, $X L$ - extra-large size eggs 
In Fig. 8, the normalized mean values (i.e. relative to the corresponding shell projection area) of the air chamber projection areas are linked to the elapsed days. The relative increment of the air chamber sizes is clearly identifiable in the front projection and, to a lesser extent, in the lateral one. The latter generally appears less representative due to the air chamber growth asymmetry, which often tends to rotate around the egg barycentric axis. As already noted by analysing the egg weight relative reduction, the air chamber projection area increment, normalized with respect to the corresponding shell projection area, shows a different trend depending on the commercial egg category. Smaller eggs exhibit a faster growth of the air chamber than larger ones. This seems to corroborate the existence of a correlation between the egg's weight reduction and the normalized air chamber areas taken either frontally or laterally.
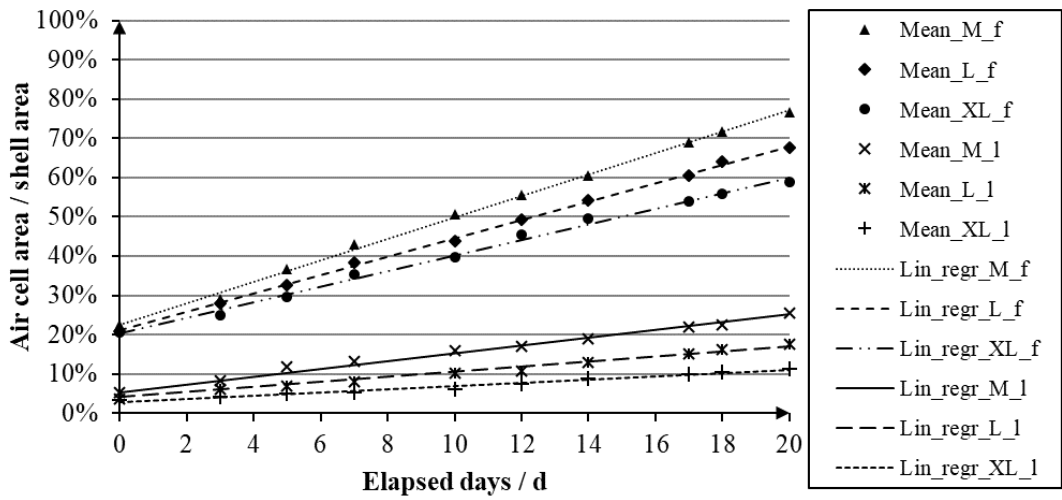

Fig. 8. Growth of the egg's normalized air chamber area: $f$ - front view; I - lateral view; $M$ - medium size eggs, $L$ - large size eggs, $X L$ - extra-large size eggs

To support this last statement, in Fig. 9 average values of the normalized air chamber projection areas relative to the mean egg weight have been plotted against the elapsed days. The trend charts show a marked linearity $\left(R^{2}>0.998\right)$ and remain distinct from each other without superpositions.
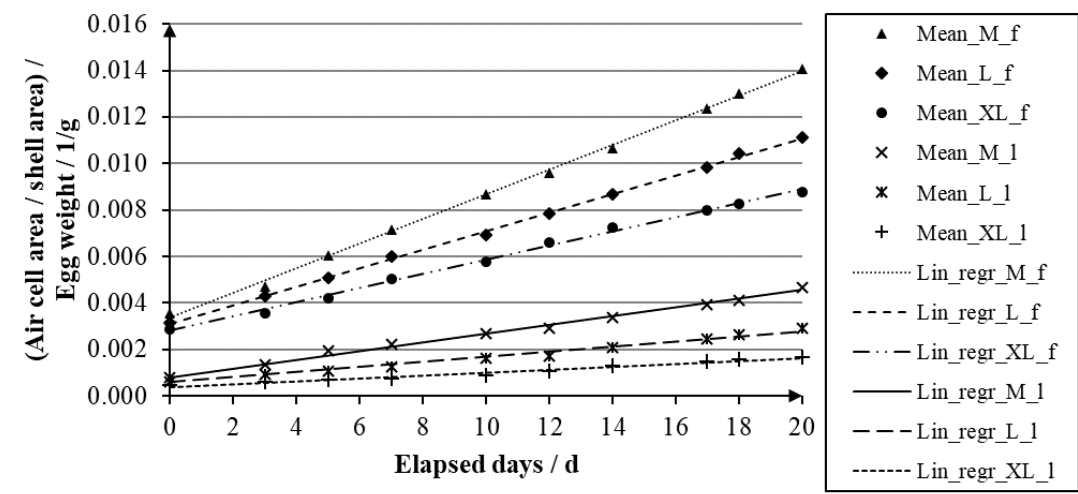

Fig. 9. Average values of normalized air chamber projection areas relative to the mean egg weight against aging time

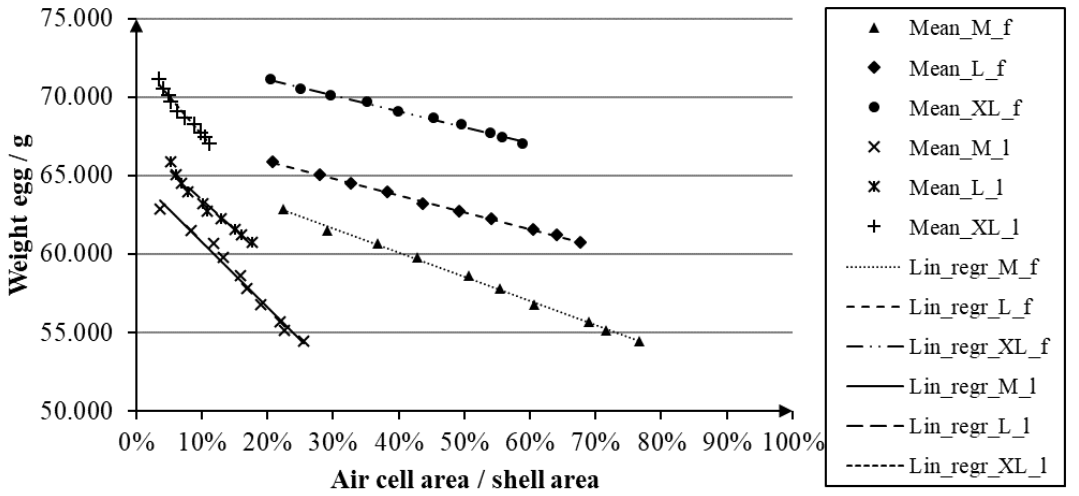

Fig. 10. Egg's weight as a function of the normalized egg's air chamber projection area 


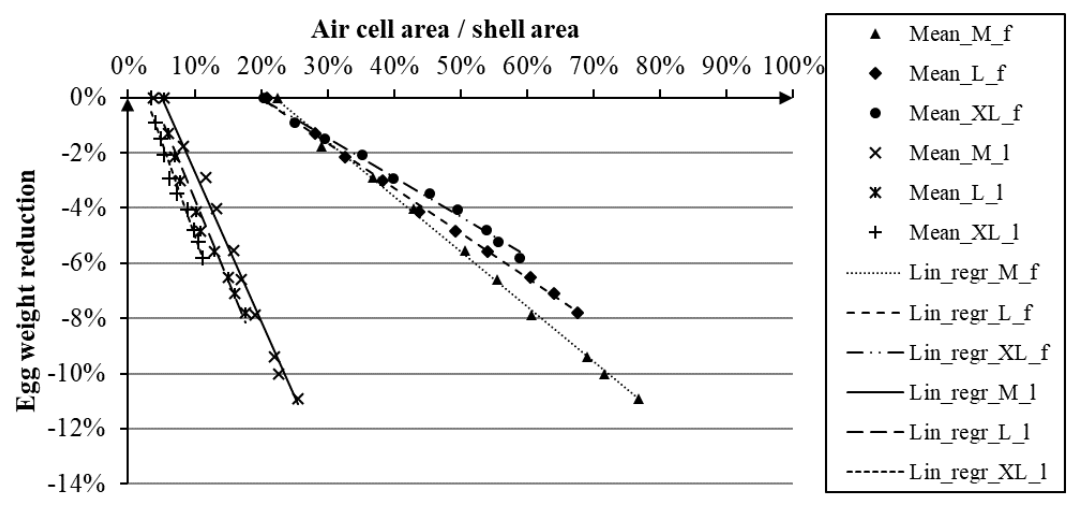

Fig. 11. Relative weight loss as a function of the normalized egg's air chamber projection area

Figs. 10 and 11 show, respectively, the weight and the relative weight loss as a function of the normalized projection area of egg's air cell. The statistical regressions actually show slightly different slopes depending on the category of egg classification. However, it should be emphasized that the sampling used has a rather limited number and further tests are needed to demonstrate the statistical significance of the observed trend.

Finally, Figure 12 shows the aggregated, non-categorized data. In this case, clearly, the statistical dispersion increases, but it is nevertheless possible to recognize a linear correlation between the relative weight loss and the normalized projection areas of the egg's air chamber, regardless of the class to which it belongs.

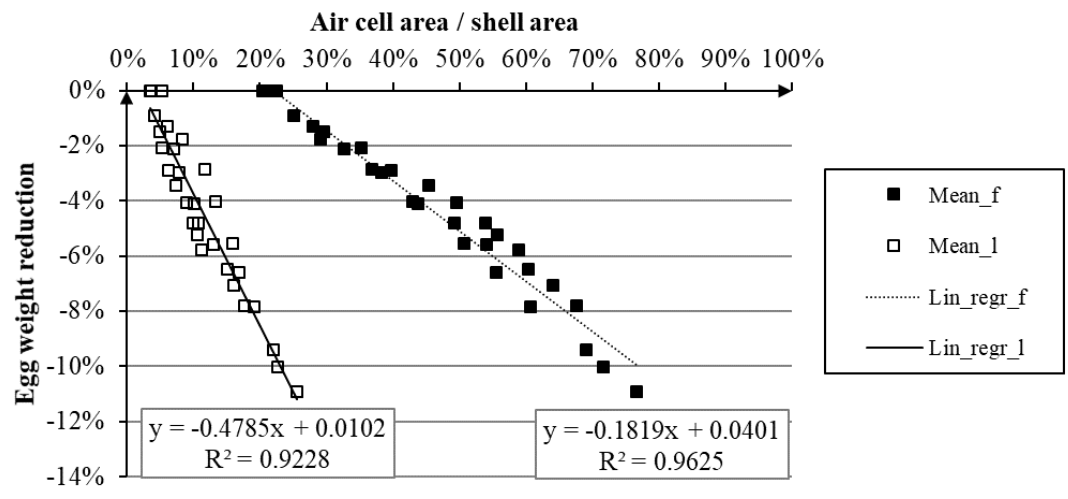

Fig. 12. Average aggregated non-categorized data of relative weight loss as a function of the normalized egg's air chamber projection area

\section{Conclusions}

In recent decades, the increasing attention to the quality of food products has developed the need for more precise and automated quality controls. Their ultimate aim is to ensure food safety and the maintenance of adequate and uniform organoleptic characteristics for the individual products offered for sale. In this context, this paper examines the problem of evaluating the conservation status of eggs for human consumption. This has been carried out through the development of an original approach based on the use of pulsed thermography and the subsequent automatic processing of the thermograms, using advanced image processing techniques aimed at quantitative analysis.

The preliminary results presented in this work, based on a limited sampling, showed a good correlation between infrared imaging of egg's air chamber projection areas and the weight loss, the latter associated with dehydration and consequent microbial contamination of the albumen. Moreover, a systematic dependence of the speed of aging from the dimensional class of eggs has been highlighted. Future researches will be focused on multiple directions, suggested by the critical analysis of the first results obtained. In particular, these points of interest will be addressed:

- experimentation on a most representative sampling (at least 120 eggs), homogeneous and robust (purchase directly from the producer);

- $\quad$ study of the effect of environmental variables (relative humidity and temperature) on the egg preservation status;

- $\quad$ estimation of the egg's air chamber volume from the measurement of the projection areas;

- $\quad$ relationship between the air chamber growth and the organoleptic egg properties measured by electronic nose; 
- $\quad$ use of raw IR phase images obtained through DFT on the sequence of scanned raw images;

- $\quad$ refinement of the automatic raw IR images processing;

- $\quad$ implementation of prediction models based on neural networks.

\section{REFERENCES}

[1] Gazzetta Ufficiale della Repubblica Italiana n¹11 del 14-05-2010 - Modalità per l'applicazione di disposizioni comunitarie in materia di applicazione della commercializzazione delle uova.

[2] Regolamento (CE) N. 589/2008 della Commissione della Comunità Europea del 23 giugno 2008 recante modalità di applicazione del regolamento (CEE) n. 1234 /2007 del Consiglio per quanto riguarda le norme di commercializzazione applicabili alle uova

[3] Brake, J., Walsh, T. J., Benton Jr, C. E., Petitte, J. N., Meijerhof, R., \& Penalva, G. (1997). Egg handling and storage. Poultry Science, 76(1), 144-151.

[4] Toussant, M. J., \& Latshaw, J. D. (1999). Ovomucin content and composition in chicken eggs with different interior quality. Journal of the Science of Food and Agriculture, 79(12), 1666-1670.

[5] Lastinger, A. W., \& McLendon, B. D. (1976). Evaluation of light transmission as a measure of egg fertility. ASAE Pape, (76-3032).

[6] Jacob, J. P., Miles, R. D., \& Mather, F. B. (2000). Egg quality. Cooperative Extension Service, Institute of Food and Agricultural Sciences (IFAS), University of Florida PS24.

[7] De Ketelaere, B., Bamelis, F., Kemps, B., Decuypere, E., \& De Baerdemaeker, J. (2004). Non-destructive measurements of the egg quality. World's Poultry Science Journal, 60(03), 289-30

[8] Kemps, B. J., Bamelis, F. R., De Ketelaere, B., Mertens, K., Tona, K., Decuypere, E. M., \& De Baerdemaeker, J. G. (2006). Visible transmission spectroscopy for the assessment of egg freshness. Journal of the Science of Food and Agriculture, 86(9), 1399-1406.

[9] Schmilovitch, Z., Hofmann, A., Egozi, H., \& Klein, E. (2002). Determination of egg freshness by NNIRS (nearnear infrared spectroscopy). In Proceedings of the AgEng Conference, Budapest, Hungary.

[10] Karoui, R., Mazerolles, G., \& Dufour, É. (2003). Spectroscopic techniques coupled with chemometric tools for structure and texture determinations in dairy products. International Dairy Journal, 13(8), 607-620.

[11] Safar, M., Bertrand, D., Robert, P., Devaux, M. F., \& Genot, C. (1994). Characterization of edible oils, butters and margarines by Fourier transform infrared spectroscopy with attenuated total reflectance. Journal of the American Oil Chemists' Society, 71(4), 371-377.

[12] Posudin YI (1998). Lasers in agriculture. Science Publishers, New York

[13] Giunchi, A., Berardinelli, A., Ragni, L., Fabbri, A., \& Silaghi, F. A. (2008). Non-destructive freshness assessment of shell eggs using FT-NIR spectroscopy. Journal of food engineering, 89(2), 142-148.

[14] Karoui, R., Kemps, B., Bamelis, F., De Ketelaere, B., Decuypere, E., \& De Baerdemaeker, J. (2006). Methods to evaluate egg freshness in research and industry: A review. European Food Research and Technology, 222(5-6), 727-732.

[15] Völgyi, F. (2000). Specifying the freshness of eggs using microwave sensors. Subsurface Sensing Technologies and Applications, 1(1), 119-139.

[16] Dutta, R., Hines, E. L., Gardner, J. W., Udrea, D. D., \& Boilot, P. (2003). Non-destructive egg freshness determination: an electronic nose based approach. Measurement Science and Technology, 14(2), 190.

[17] Yongwei, W., Wang, J., Zhou, B., \& Lu, Q. (2009). Monitoring storage time and quality attribute of egg based on electronic nose. Analytica Chimica Acta, 650(2), 183-188.

[18] Aboonajmi, M., Setarehdan, S. K., Akram, A., Nishizu, T., \& Kondo, N. (2014). Prediction of poultry egg freshness using ultrasound. International Journal of Food Properties, 17(9), 1889-1899.

[19] Montanini, R., Quattrocchi, A., \& Piccolo, S. A. (2016). Active thermography and post-processing image enhancement for recovering of abraded and paint-covered alphanumeric identification marks. Infrared Physics \& Technology, 78, 24-30.

[20] Montanini, R. (2010). Quantitative determination of subsurface defects in a reference specimen made of Plexiglas by means of lock-in and pulse phase infrared thermography. Infrared Physics \& Technology, 53 (5), 363-71. 